Rafael Nogueira Scoriza ${ }^{1 *}$

Maria Elizabeth Fernandes Correia ${ }^{2}$

Eliane Maria Ribeiro da Silva ${ }^{2}$

${ }^{1}$ Universidade Federal Rural do Rio de Janeiro

- UFRRJ, Rodovia BR 465, Km 07, s/n, Zona

Rural, 23890-000, Seropédica, RJ, Brasil

${ }^{2}$ Empresa Brasileira de Pesquisa Agropecuária -

Embrapa Agrobiologia, Rodovia BR 465, Km 07,

s/n, Zona Rural, 23890-000, Seropédica, RJ,

Brasil

${ }^{*}$ Autor Correspondente:

E-mail: rafaelscoriza@gmail.com

PALAVRAS-CHAVE

Comunidade biológica

Conservação florestal

Bioindicador

\section{KEYWORDS}

Biological community

Forest conservation

Bioindicator

\title{
Colêmbolos e fungos micorrízicos arbusculares como indicadores de degradação em fragmentos florestais de encosta
}

\author{
Springtails and mycorrhizal fungi as indicators of \\ degradation in forest fragments of slope
}

RESUMO: Para o sucesso de qualquer medida de conservação e recuperação, é necessário avaliar e interpretar o grau de degradação das florestas fragmentadas, ambiente dominante na paisagem tropical. Com isso, o objetivo deste trabalho foi avaliar os colêmbolos epígeos e os fungos micorrízicos arbusculares (FMA) como indicadores de degradação em fragmentos florestais de encostas de morrotes. O estudo foi realizado em quatro fragmentos florestais nas bacias hidrográficas dos rios Macacu e Caceribu, no município de Itaboraí, Estado do Rio de Janeiro. Como os fragmentos florestais estão em morrotes, as coletas foram realizadas nos terços superior, médio e inferior, na época seca e úmida do ano. Na comunidade de colêmbolos, a ordem Entomobryomorpha foi predominante, com maior número de indivíduos na época seca. Para os FMA, a menor densidade de esporos no fragmento P1 é um indicativo de uma biota mais diversa e estável. Ambas as comunidades apresentaram relação com atributos do solo e da vegetação, denotando sua sensibilidade a fatores e a alterações ambientais. Dessa forma, mesmo ocupando diferentes estratos verticais do solo, apresentaram respostas semelhantes, apontando o fragmento florestal P1 como o mais conservado e com potencial de ser utilizado como referência em projetos de recuperação de áreas degradadas.

\begin{abstract}
Fragmented forests is the dominant environment in the tropical landscape, and to the success of any conservation and recovery; it is necessary to evaluate and interpret the degree of degradation of these fragments. Thus, this study aimed to evaluate the springtails epigean and mycorrhizal fungi as indicators of degradation in forest fragments slopes of hillocks. The study was conducted in four forest fragments in the basins of rivers Macacu and Caceribu, Itaborai (Rio de Janeiro state). As forest fragments are in hillocks, collections were made in the upper, middle and lower thirds, in dry and wet seasons of year. In the springtail community the order Entomobryomorph a was predominant, with greater number of individuals in the dry season. For mycorrhizal fungi, the lower spore density in the P1 fragment is indicative of a more diverse and stable biota. Both communities presented a relationship with soil and vegetation attributes, denoting their sensitivity to environmental factors and changes. Thus, even though they occupy different vertical strata of the soil, they presented similar responses, indicating the P1 forest fragment as the most conserved and with potential to be used as a reference in degraded area recovery projects.
\end{abstract}




\section{Introdução}

Um longo histórico de uso do solo nas bacias hidrográficas dos rios Macacu e Caceribu, no município de Itaboraí, Estado do Rio de Janeiro, com a cultura canavieira, fruticultura, pastagens, extração de areia e de argila, transformou toda a paisagem da região, criando fragmentos florestais de tamanho e de grau de degradação diversificado (Embrapa, 2007). Esse cenário retrata as estimativas do Instituto Nacional de Pesquisas Espaciais e da Fundação SOS Mata Atlântica (2013) de que apenas 18,6\% são vegetação original para o Estado. Mesmo com capacidade limitada de reter espécies e de prover serviços ambientais, esse é o ambiente dominante e alvo de conservação nas regiões tropicais (Tabarelli et al., 2012).

Para a aplicação de qualquer medida que vise ao restabelecimento da vegetação e de seus processos naturais, é necessário avaliar e interpretar os impactos naturais ou antrópicos a partir de informações obtidas das propriedades ou dos processos biológicos no solo (Ferreira Júnior et al., 2012). Os organismos ali presentes são uma importante ferramenta para avaliar e interpretar os efeitos de interferências naturais ou antrópicas (Heger et al., 2012), pois estão integrados e relacionados a funções ecossistêmicas (Muscolo et al., 2014).

O compartimento formado pela serrapilheira e o solo superficial fornecem carbono, energia e abrigo para grande parte dos organismos do solo, que são responsáveis por processos biológicos e físico-químicos essenciais para o desenvolvimento e funcionalidade do ecossistema terrestre, como a decomposição e a ciclagem de nutrientes (Jeffery et al., 2010). Dentre esses organismos, os colêmbolos ocupam diversos estratos verticais do solo (Chauvat et al., 2014), onde exercem um grande papel na formação da microestrutura do solo, na fragmentação de excrementos e da serrapilheira, e na ciclagem de nutrientes por meio de herbivoria e de interações com os microrganismos decompositores. Geralmente, são indicadores sensíveis de distúrbios e importantes na avaliação ambiental (Greenslade, 1991; Rusek, 1998).

Outro grupo de organismos de grande importância são os fungos micorrízicos arbusculares (FMA), capazes de formar associação simbiótica com a maioria das plantas (Smith \& Read, 2008). Eles desempenham importante papel na ciclagem do carbono e do fósforo, no aumento da estabilidade e da estruturação do solo por intermédio da criação de micro e macroagregados e, consequentemente, na retenção de água (Daynes et al., 2013; Peng et al., 2013). Ocorrem de forma generalizada mesmo em ambientes com avançado grau de degradação e são muito sensíveis às interferências no ecossistema, considerado como um importante parâmetro biológico para avaliar o distúrbio do ambiente em áreas de Floresta Atlântica (Pereira et al., 2014).

Com isso, o objetivo deste trabalho foi utilizar as comunidades biológicas do solo de colêmbolos epígeos e dos FMA, que possuem distintos habitats verticais, como indicadoras de degradação em fragmentos florestais de encostas de morrotes.

\section{Material e Métodos}

A área de estudo está inserido em meio a morrotes de pequena envergadura do terciário e proterozoico, nas bacias hidrográficas dos rios Macacu e Caceribu, entre as coordenadas $42^{\circ} 47^{\prime} 35^{\prime \prime} \mathrm{W} / 42^{\circ} 49^{\prime} 20^{\prime \prime} \mathrm{W}$ e $22^{\circ} 40^{\prime} 35^{\prime} \mathrm{S} / 22^{\circ} 38^{\prime} 51 \mathrm{~S}$. Parte dessas bacias está inserida em um complexo de unidades de conservação, que é composto pelo Parque Nacional das Serras dos Órgãos, Estação Ecológica Estadual do Paraíso e Áreas de Proteção Ambiental (Guapimirim, Petrópolis, do Rio São João/ Mico-Leão-Dourado, entre outras).

Nos compartimentos ambientais mais altos, há a predominância do Cambissolo e do Argissolo Amarelo, enquanto nos compartimentos de planície, dos Gleissolos e dos Neossolos Flúvicos (Embrapa, 2007). A classificação climática, segundo Koppen, é Aw, ou seja, clima tropical úmido ou subúmido, com inverno seco. A temperatura média anual é de $21,4{ }^{\circ} \mathrm{C}$, com precipitação média anual de $1.460 \mathrm{~mm}$ (dezembro é o mês mais chuvoso). Os fragmentos são domínios da Floresta Ombrófila Densa de Terras Baixas e Submontana. A característica da paisagem na área é uma matriz de pastagens, com predomínio das espécies de Brachiaria humidicola, Brachiaria decumbens e Paspalum sp.

As amostragens foram realizadas em quatro fragmentos florestais, variáveis em tamanho: T1 com uma área de 7 ha, T2 com 3 ha, P1 com 21 ha e P2 com 10 ha. Como os fragmentos florestais estão sob encostas, eles foram divididos nos terços superior, médio e inferior. Realizaram-se, em cada terço, coletas de fauna epígea do solo - onde se avaliou o grupo Collembola - e de amostras de solo para avaliação de FMA. As coletas ocorreram em dois momentos: no final da época seca (junho/2010) e no final da época úmida (fevereiro/2011).

A avaliação da comunidade de Collembola foi realizada por meio de armadilhas do tipo pitfall, isto é, recipientes plásticos com $9 \mathrm{~cm}$ de diâmetro e $11 \mathrm{~cm}$ de altura, com líquido preservativo (formalina a 4\%), enterrados no solo com a borda no nível da superfície, segundo a metodologia de Aquino et al. (2006). Foram instaladas seis armadilhas em linha, em cada um dos terços, distando $5 \mathrm{~m}$ entre si, que permaneceram no local por sete dias. Em laboratório, o conteúdo dos recipientes foi transferido para frascos contendo álcool a 70\%, com a finalidade de preservação em longo prazo. Os indivíduos foram identificados entre as ordens Entomobryomorpha, Poduromorpha e Symphypleona, de acordo com as características morfológicas (Zeppelini Filho \& Bellini, 2004).

Para a avaliação da comunidade de FMA, coletaram-se seis amostras simples de solo na profundidade de $0-5 \mathrm{~cm}$ em cada terço dos fragmentos. De cada amostra, foram pesados $50 \mathrm{~g}$ de solo para as extrações dos esporos dos FMA, segundo a técnica adaptada de peneiramento úmido (Gerdemann \& Nicolson, 1963). A contagem foi processada em placa de Petri canelada sob microscópio estereoscópico. Para a identificação os esporos, colocaram-se em lâminas com álcool polivinil em lactoglicerol (PVLG) sob uma lamínula. Na mesma lâmina, um segundo grupo de esporos foi submetido a uma mistura de PVLG + Reagente de Melzer (1:1) e quebrados mecanicamente, sob uma lamínula, para exposição das paredes internas. A reação de cor ao reagente de Melzer foi utilizada para caracterizar as paredes dos esporos. A identificação das espécies de FMA foi feita segundo Schenck \& Perez (1988), e a descrição morfológica, por meio de informações disponíveis na internet, na página da International Culture Collection of Arbuscular Mycorrhizal Fungi (INVAM, 2015).

Para caracterizar e contrastar o ambiente com os dados das comunidades biológicas avaliadas, foram utilizados dados 
dos atributos do solo (Gomes et al., 2014) e da vegetação (Uhlmann et al., 2012), obtidos nas mesmas épocas e locais, como apresentados na Tabela 1.

O número de esporos de FMA foram estimados para a densidade esporos $\mathrm{g}^{-1}$ de solo. Além disso, avaliou-se a ocorrência relativa de espécies em relação aos fragmentos, utilizando como unidade as repetições. Os dados da contagem nas ordens de colêmbolos resultantes de cada armadilha foram estimados em indivíduos armadilha ${ }^{-1}$ dia $^{-1}$ (atividade). Para a análise dos resultados, realizou-se a avaliação da homogeneidade das variâncias dos erros pelo teste de Cochran e da normalidade pelo teste de Lilliefors. Posteriormente, os dados paramétricos foram submetidos à análise de variância com aplicação do teste $\mathrm{t}$ (Bonferroni) a 5\% de probabilidade, enquanto os não paramétricos, pelos testes de Wilcoxon (para a comparação entre períodos amostrais) e Kruscal-Wallis (para a comparação entre terços e fragmentos). As análises de correlação de Pearson (paramétricos) ou de Spearman (não paramétricos) a $5 \%$, conforme o tipo de dado, relacionam as comunidades biológicas com os dados ambientais.

\section{Resultados e Discussão}

Considerando a soma de todos os fragmentos, a atividade de colêmbolos na época seca foi de 957,84 indivíduos armadilha $^{-1}$ dia $^{-1}$, dos quais $74,4 \%$ eram representados por Entomobryomorpha, 18\%, por Poduromorpha, e 7,6\%, por

Tabela 1. Fertilidade, granulometria e física do solo, vegetação e outras características dos terços (divisão da encosta) dos fragmentos florestais de encosta em Itaboraí, no Estado do Rio de Janeiro.

Table 1. Fertility, granulometry and physical soil, vegetation and other features of thirds (division of the slope) of forest fragments slope in Itaboraí, RJ.

\begin{tabular}{|c|c|c|c|c|}
\hline Atributo & $\mathrm{P} 1$ & $\mathrm{P} 2$ & T1 & $\mathrm{T} 2$ \\
\hline & \multicolumn{4}{|c|}{ Fertilidade do solo } \\
\hline $\mathrm{pH}$ & 5,3 & 5,6 & 4,9 & 5,6 \\
\hline $\mathrm{Al}^{+3}$ & 1,6 & 1,3 & 3,1 & 1,3 \\
\hline $\mathrm{Ca}^{+2}$ & 0,4 & 0,4 & 0,4 & 0,6 \\
\hline $\mathrm{Mg}^{+2}$ & 0,2 & 0,4 & 0,2 & 0,1 \\
\hline $\mathrm{K}^{+}$ & 0,05 & 0,09 & 0,07 & 0,03 \\
\hline $\mathrm{P}$ & 2,0 & 3,0 & 2,6 & 1,6 \\
\hline \multirow[t]{2}{*}{$\mathrm{C}$} & 7,2 & 15,9 & 14,9 & 6,6 \\
\hline & \multicolumn{4}{|c|}{ Características físicas do solo } \\
\hline Areia grossa & 326 & 431 & 369 & 443 \\
\hline Areia fina & 146 & 101 & 143 & 216 \\
\hline Silte & 129 & 127 & 55 & 66 \\
\hline Argila & 400 & 342 & 433 & 275 \\
\hline Densidade do solo & 1,10 & 1,27 & 1,53 & 1,21 \\
\hline \multirow[t]{2}{*}{ Porosidade } & 0,60 & 0,50 & 0,45 & 0,54 \\
\hline & \multicolumn{4}{|c|}{ Vegetação } \\
\hline Número de espécies & 14 & 5 & 7 & 16 \\
\hline Dominância & 1,00 & 0,28 & 0,36 & 0,94 \\
\hline Densidade & 3000 & 1317 & 1300 & 1600 \\
\hline
\end{tabular}

P1, P2, T1 e T2 são fragmentos florestais com 21, 10, 7 e 3 hectares, respectivamente; Unidades: $\mathrm{pH}=\mathrm{CaCl}_{2} ; \mathrm{Al}^{3+}, \mathrm{Ca}^{2+}, \mathrm{Mg}^{2+} \mathrm{e} \mathrm{K}^{+}=\mathrm{cmol}_{\mathrm{c}} \mathrm{dm}^{-3}$; $\mathrm{P}=\mathrm{mg} \mathrm{dm}{ }^{-3} ; \mathrm{C}=\mathrm{g} \mathrm{dm}^{-3} ;$ Areia grossa, Areia fina, Silte e Argila $=\mathrm{g} \mathrm{kg}^{-1}$; Densidade do solo $=\mathrm{g} \mathrm{cm}^{-3}$; Porosidade $=\%$; Dominância $=\mathrm{m}^{2} \mathrm{ha}^{-1}$; Densidade $=$ Ind. ha ${ }^{-1}$.
Symphyplenona. Na época úmida, a atividade foi inferior à época seca, com 425,38 indivíduos ha $\mathrm{hi}^{-1} \mathrm{dia}^{-1}$, dos quais $98 \%$ eram de Entomobryomorpha, 0,1\%, de Poduromorpha, e 1,9\%, de Symphypleona. Essa predominância de Entomobryomorpha em ambas as coletas pode estar relacionada ao método passivo de coleta utilizado, já que essa ordem é a mais ativa e apresenta maior habilidade de dispersão e salto, quando comparada à de Poduromorpha e de Symphypleona (Hopkin, 1997).

Em relação à sazonalidade, houve elevada atividade na época seca do ano nos fragmentos florestais. Uma possível explicação para esse resultado também está relacionada ao método de coleta, visto que se utiliza um líquido no recipiente. Especificamente para a ordem Entomobryomorpha, a maioria das espécies possui elevada capacidade de habitar ambientes mais secos, pois seus corpos são cobertos por abundantes cerdas e escamas, as quais retêm uma camada de ar "aprisionado" sobre o corpo e diminui a perda de água (Zeppelini Filho \& Bellini, 2004).

A comunidade de colêmbolos foi mais eficiente em indicar a diferença entre os fragmentos florestais do que entre os terços da encosta. Na época seca, o fragmento T1 apresentou maior atividade, com superioridade do fragmento $\mathrm{P} 1$ para a ordem Entomobryomorpha e dos fragmentos P2 e T2 para as ordens Poduromorpha e Symphypleona. Já na época úmida, a atividade no fragmento T1 esteve entre as menores (Tabela 2).

A divisão da encosta do morrote em terços mostrou pouca relação com a atividade dos colêmbolos, pois foram observadas diferenças pontuais entre esses terços. O único caso com algum padrão foi verificado no fragmento P2 durante a época úmida, quando o terço inferior apresentou maior atividade que o terço médio para as ordens Entomobryomorpha e Symphypleona (Tabela 2).

Esses resultados mostram que há diferenças entre os fragmentos florestais avaliados e que as ordens de colêmbolos considerados apresentam distintas sensibilidades, alteradas pelas condições climáticas. Considerando que esses organismos estão intimamente ligados às funções ambientais, já eram esperadas variações em sua densidade entre ecossistemas afetados por atividades antrópicas (Rusek, 1998) modificadoras da composição vegetal e de parâmetros do solo.

De maneira semelhante à comunidade de colêmbolos, não houve diferenças entre os terços da encosta para a densidade média de esporos de FMA. Comparando os fragmentos na época seca, verificou-se uma menor densidade de esporos no fragmento P1 (44,2 esporos. $\mathrm{g}^{-1}$ de solo) na comparação com os demais. Na época úmida, o fragmento P2 (46,7 esporos. $\mathrm{g}^{-1} \mathrm{de}$ solo) apresentou maior densidade de esporos que o fragmento T2 (29,6 esporos. $\mathrm{g}^{-1}$ de solo) e P1 (22,8 esporos. $\mathrm{g}^{-1}$ de solo) (Figura 1).

Segundo Zangaro \& Moreira (2010), a abundância dos esporos e a colonização das raízes por FMA, de maneira geral, têm sido maior nos ambientes que se encontram nos estádios iniciais da sucessão do que nos ambientes com sucessão mais avançada e na floresta clímax. A menor densidade de esporos de FMA e a maior riqueza de espécies, encontradas nas áreas mais conservadas, podem ser consequência da sua maior estabilidade e menor competição por nichos (guildas), dando garantia da sobrevivência das espécies com baixa esporulação. Pode-se considerar também a presença de uma biota micófaga mais ativa 
Tabela 2. Atividade das ordens de colêmbolos nos terços (divisão da encosta) e nos fragmentos florestais de encosta, em Itaboraí, no Estado do Rio de Janeiro.

Table 2. Activity of springtails orders in thirds and forest fragments thirds (division of the slope), in Itaboraí, RJ.

\begin{tabular}{|c|c|c|c|c|c|c|c|}
\hline \multirow{2}{*}{ Fragmento } & \multirow{2}{*}{ Terço } & Ent. ${ }^{(2)}$ & Pod..$^{(3)}$ & Sym. ${ }^{(4)}$ & Ent. & Pod. & Sym. \\
\hline & & \multicolumn{3}{|c|}{ Época seca } & \multicolumn{3}{|c|}{ Época úmida } \\
\hline \multirow{4}{*}{ P1 } & Superior & $7,0 \mathrm{a}$ & 1,9 a & $1,1 \mathrm{a}$ & $12,3 \mathrm{a}$ & 0,0 & 0,0 \\
\hline & Médio & $5,6 \mathrm{a}$ & $4,5 \mathrm{a}$ & $1,1 \mathrm{a}$ & $15,1 \mathrm{a}$ & 0,0 & 0,0 \\
\hline & Inferior & $3,5 \mathrm{a}$ & $2,9 \mathrm{a}$ & $1,0 \mathrm{a}$ & $7,5 \mathrm{a}$ & 0,0 & 0,1 \\
\hline & Média & $5,4 \mathrm{~B}$ & $3,1 \mathrm{AB}$ & $1,1 \mathrm{AB}$ & $11,6 \mathrm{~A}$ & 0,0 & $0,0 \mathrm{~B}$ \\
\hline \multirow{4}{*}{$\mathrm{P} 2$} & Superior & $8,5 \mathrm{a}$ & $1,7 \mathrm{a}$ & $0,6 \mathrm{a}$ & $1,6 \mathrm{~b}$ & 0,0 & $0,2 \mathrm{ab}$ \\
\hline & Médio & $9,2 \mathrm{a}$ & $1,4 \mathrm{a}$ & $0,5 \mathrm{a}$ & $0,9 \mathrm{~b}$ & 0,0 & $0,1 \mathrm{~b}$ \\
\hline & Inferior & $6,7 \mathrm{a}$ & $1,5 \mathrm{a}$ & $0,7 \mathrm{a}$ & $3,4 \mathrm{a}$ & 0,0 & $0,5 \mathrm{a}$ \\
\hline & Média & $8,2 \mathrm{AB}$ & $1,6 \mathrm{BC}$ & $0,6 \mathrm{~B}$ & $2,0 \mathrm{C}$ & 0,0 & $0,3 \mathrm{~A}$ \\
\hline \multirow{4}{*}{$\mathrm{T} 1$} & Superior & $6,8 \mathrm{a}$ & $4,9 \mathrm{a}$ & $0,9 \mathrm{a}$ & 3,4 & 0,0 & 0,0 \\
\hline & Médio & $20,0 \mathrm{a}$ & $1,6 \mathrm{a}$ & $1,6 \mathrm{a}$ & - & - & - \\
\hline & Inferior & $16,9 \mathrm{a}$ & $4,8 \mathrm{a}$ & $2,6 \mathrm{a}$ & 2,6 & 0,0 & 0,1 \\
\hline & Média & $14,5 \mathrm{~A}$ & $3,8 \mathrm{~A}$ & $1,7 \mathrm{~A}$ & $3,0 \mathrm{C}$ & 0,0 & $0,1 \mathrm{~B}$ \\
\hline \multirow{4}{*}{$\mathrm{T} 2$} & Superior & $11,0 \mathrm{a}$ & $0,7 \mathrm{a}$ & $0,6 \mathrm{ab}$ & $6,2 \mathrm{a}$ & 0,0 & 0,0 \\
\hline & Médio & $10,5 \mathrm{a}$ & $1,6 \mathrm{a}$ & $1,0 \mathrm{a}$ & $9,0 \mathrm{a}$ & 0,0 & 0,0 \\
\hline & Inferior & $13,2 \mathrm{a}$ & $1,1 \mathrm{a}$ & $0,3 \mathrm{~b}$ & $7,5 \mathrm{a}$ & 0,0 & 0,3 \\
\hline & Média & $11,5 \mathrm{AB}$ & $1,1 \mathrm{C}$ & $0,7 \mathrm{~B}$ & $7,6 \mathrm{~B}$ & 0,0 & $0,1 \mathrm{~B}$ \\
\hline
\end{tabular}

P1, P2, T1 e T2 são fragmentos florestais com 21, 10, 7 e 3 hectares, respectivamente; Letras iguais minúsculas (comparação entre terços do mesmo fragmento) e maiúsculas (comparação entre fragmentos) não diferem pelo teste de Kruskal-Wallis a $5 \%{ }^{(2)}=$ Entomobryomorpha; ${ }^{(3)}=$ Poduromorpha; ${ }^{(4)}=$ Symphypleona.

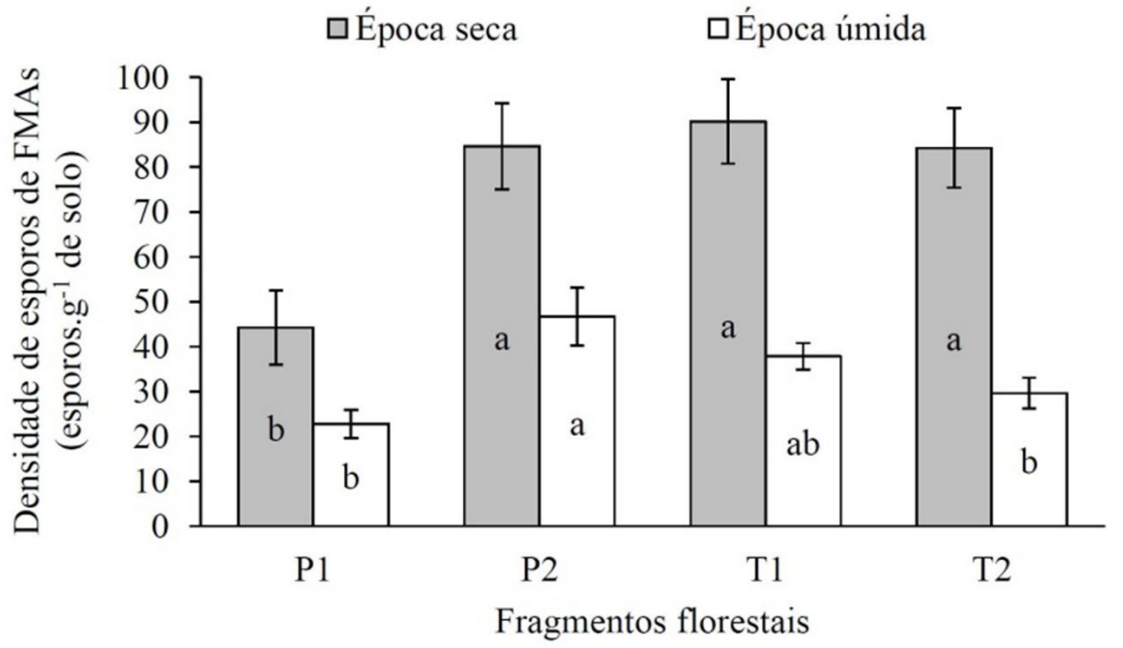

Figura 1. Densidade de esporos nos fragmentos florestais, nos períodos seco e úmido. Letras iguais na mesma época não diferem segundo o teste t (Bonferroni) a $5 \%$. Fonte: os autores.

Figure 1. Density of spores in forest fragments in the dry and wet periods. Letters equal the same time do not differ according to the $t$ test (Bonferroni) to $5 \%$. Source: authors.

ou a predominância de espécies não pioneiras (espécies K), que não induzem grandes esporulações (Pereira et al., 2014). Bonfim et al. (2013) relacionam o menor número de esporos ao maior investimento na produção de hifas, ao invés de esporos, nos ambientes florestais mais bem estabelecidos. Com essas afirmações e contrastando os dados deste trabalho com os trabalhos citados anteriormente, podemos inferir que o fragmento florestal P1 é o mais conservado.

Entretanto, para a riqueza de espécies, essa constatação é confirmada apenas na época seca, quando foram identificadas 14 espécies no fragmento P1, 11 em P2 e 13 em T1 e T2. Já na época úmida, foram identificadas 9 espécies nos fragmentos P1 e P2, 10 em T1 e 11 em T2 (Tabela 3).

Uma alta riqueza de espécies de FMA está relacionada a áreas mais conservadas, com condições ambientais adequadas e com uma maior diversidade de plantas hospedeiras favoráveis (Zangaro \& Moreira, 2010; Deepika \& Kothamasi, 2014), conceituação esta que pode ser aplicada novamente ao fragmento P1, na época seca. Além disso, algumas espécies de baixa frequência foram encontradas apenas nesse fragmento, como é o caso de Acaulospora tuberculata, Acaulospora undulata e Scutellospora cerradensis. De maneira contrária, 
Tabela 3. Espécies e frequência relativa (\%) de fungos micorrízicos arbusculares (FMA) encontradas nos fragmento florestais nas épocas do ano.

Table 3. Species and relative frequency (\%) of Arbuscular Mycorrhizal Fungi found in the forest fragment in seasons.

\begin{tabular}{|c|c|c|c|c|c|c|c|c|}
\hline \multirow{2}{*}{ Espécies de FMA } & \multicolumn{4}{|c|}{ Época seca } & \multicolumn{4}{|c|}{ Época úmida } \\
\hline & P1 & P2 & T1 & $\mathrm{T} 2$ & P1 & P2 & $\mathrm{T} 1$ & $\mathrm{~T} 2$ \\
\hline Acaulospora foveata $^{(1)}$ & 28 & 0 & 39 & 17 & 61 & 44 & 33 & 33 \\
\hline Acaulospora laevis ${ }^{(2)}$ & 39 & 28 & 33 & 22 & 39 & 78 & 44 & 61 \\
\hline Acaulospora mellea $^{(3)}$ & 56 & 56 & 56 & 50 & 33 & 22 & 28 & 0 \\
\hline Acaulospora scrobiculata $^{(4)}$ & 6 & 0 & 6 & 0 & 0 & 0 & 0 & 0 \\
\hline Acaulospora tuberculata $^{(5)}$ & 6 & 0 & 0 & 0 & 0 & 0 & 0 & 0 \\
\hline Acaulospora undulata $^{(6)}$ & 6 & 0 & 0 & 0 & 0 & 0 & 0 & 0 \\
\hline Acaulospora sp. & 11 & 0 & 0 & 6 & 0 & 0 & 0 & 6 \\
\hline Archaeospora leptoticha(7) & 17 & 11 & 22 & 6 & 39 & 44 & 56 & 39 \\
\hline Entrophospora colombiana $^{(8)}$ & 0 & 6 & 22 & 0 & 0 & 0 & 0 & 0 \\
\hline Entrophospora sp. & 6 & 6 & 11 & 11 & 0 & 0 & 0 & 0 \\
\hline Gigaspora sp. & 22 & 6 & 0 & 22 & 39 & 11 & 28 & 33 \\
\hline Glomus etunicatum $^{(8)}$ & 0 & 0 & 0 & 0 & 0 & 0 & 0 & 6 \\
\hline Glomus lamellosum $^{(9)}$ & 0 & 44 & 22 & 28 & 0 & 11 & 0 & 0 \\
\hline Glomus macrocarpum $^{(10)}$ & 100 & 100 & 100 & 100 & 100 & 100 & 100 & 100 \\
\hline Glomus microcarpum $^{(10)}$ & 0 & 0 & 0 & 0 & 17 & 0 & 6 & 6 \\
\hline Glomus tenebrosum $^{(11)}$ & 0 & 0 & 0 & 0 & 22 & 6 & 11 & 11 \\
\hline Glomus tortuosum $^{(12)}$ & 0 & 0 & 17 & 6 & 0 & 0 & 0 & 6 \\
\hline Glomus sp. & 11 & 6 & 22 & 22 & 6 & 6 & 11 & 0 \\
\hline Scutellospora calospora ${ }^{(13)}$ & 0 & 6 & 0 & 6 & 0 & 0 & 0 & 0 \\
\hline Scutellospora cerradensis ${ }^{(14)}$ & 6 & 0 & 0 & 0 & 0 & 0 & 0 & 0 \\
\hline Scutellospora persica ${ }^{(15)}$ & 0 & 0 & 0 & 0 & 0 & 0 & 6 & 0 \\
\hline Scutellospora verrucosa ${ }^{(15)}$ & 0 & 6 & 11 & 6 & 0 & 0 & 0 & 6 \\
\hline Scutellospora sp. & 17 & 0 & 6 & 0 & 0 & 0 & 0 & 0 \\
\hline
\end{tabular}

P1, P2, T1 e T2 são fragmentos florestais com 21, 10, 7 e 3 hectares, respectivamente; ${ }^{(1)}=$ Trappe \& Janos; ${ }^{(2)}=$ Gerdemann $\&$ Trappe; ${ }^{(3)}=$ Spain $\&$ Schenck; ${ }^{(4)}=$ Trappe; ${ }^{(5)}=$ Janos \& Trappe; ${ }^{(6)}=$ Sieverdin; ${ }^{(7)}=\left(\right.$ Schenck \& Smith) Morton \& Redecker; ${ }^{(8)}=$ Becker \& Gerdemann; ${ }^{(9)}=$ Dalpe, Koske \& Tews; ${ }^{(10)}=$ Tulasne \& Tulasne; ${ }^{(1)}=($ Thaxter $)$ Berch; ${ }^{(12)}=$ Schenck \& Smith; ${ }^{(13)}=($ Nicol. \& Gerd. $)$ Walker \& Sanders; ${ }^{(14)}=$ Spain \& Miranda; ${ }^{(15)}=($ Koske \& Walker) Walker \& Sanders.

espécies como Acaulospora laevis, Archaeospora leptoticha e Glomus macrocarpum ocorreram em todos os fragmentos e épocas amostradas, com destaque para G. macrocarpum, com frequência relativa de $100 \%$.

Vale destacar também que algumas espécies de Acaulospora (A. scrobiculata, A tuberculata e A. undulata), Entrophospora e Scutellospora ( $S$. cerradensis e Scutellospora sp.) foram identificadas apenas na época seca, enquanto algumas espécies de Glomus (G. microcarpum e G. tenebrosum) e a Scutellospora pérsica, apenas na época úmida, o que demonstra a resposta diferenciada da comunidade às condições ambientais. Bonfim et al. (2013) também verificaram a esporulação de algumas espécies apenas na época chuvosa e de outras exclusivamente na seca.

As comunidades biológicas em estudo são direta e indiretamente influenciadas por características do ambiente - estas são a base de explicação de suas sensibilidades como indicadores. Para os colêmbolos, o número de indivíduos e de espécies está relacionado com variáveis de vegetação e com todos os fatores que afetam a disponibilidade de matéria orgânica (Zeppelini et al., 2009), já que a formação da associação micorrízica depende de fatores ambientais, fisiologia do hospedeiro, microrganismos do solo, quantidade e composição de fungos micorrízicos (Smith \& Read, 2008). Assim, foram encontradas correlações significativas dessas comunidades com a fertilidade, a granulometria do solo, a vegetação e outros fatores (Tabela 4).

Para Entomobryomorpha, durante a época seca, quanto menor a porosidade e maior a densidade do solo, maior a atividade dessa ordem. Portanto, nessa época, a maior atividade de indivíduos desse grupo pode ser um indicativo de distúrbios no ambiente. Já na época úmida, ocorre o inverso na relação com o solo e há um favorecimento de uma vegetação mais densa e diversa. Uma hipótese para essa controvérsia é que, na época seca, há predominância de espécies mais generalistas e com alta capacidade reprodutiva na comunidade de colêmbolos, enquanto que, na época úmida, estabelece-se um equilíbrio, com isso as espécies presentes estão mais associadas às condições do solo e da vegetação do local. A mesma hipótese pode ser estendida às ordens Poduromorpha e Symphypleona, por sua maior relação com o solo na época seca. Reunindo todas essas informações e contrastando com o ambiente (Tabela 1) e atividade de colêmbolos (Tabela 2), o fragmento P1 apresenta-se como o mais conservado, dentre os analisados.

Na comunidade de FMA, durante a época seca, a densidade de esporos apresentou-se relacionada negativamente à densidade e porosidade do solo e à densidade da vegetação. A riqueza de espécies apenas foi influenciada nessa época pelo $\mathrm{pH}$ e pelo alumínio. Já na época úmida, a relação resumiu-se à menor densidade de esporos com um maior número de espécies e dominância vegetal. Esses resultados, principalmente no tocante ao número de esporos, reforçam os resultados anteriores (Figura 1) de que quanto mais complexa a estrutura e a fisionomia da vegetação, menores as quantidades de esporos encontrados no solo. 
Tabela 4. Correlação entre as comunidades de colêmbolos e fungos micorrízicos arbusculares (FMA) com características do ambiente em fragmentos florestais de encosta, nas épocas seca e úmida.

Table 4. Correlation between the communities of springtail and AMF with environmental characteristics in forest fragments slope, in the dry and wet seasons.

\begin{tabular}{|c|c|c|c|c|c|c|c|c|c|c|}
\hline \multirow[b]{2}{*}{$\begin{array}{l}\text { Características } \\
\text { abióticas do ambiente }\end{array}$} & \multicolumn{5}{|c|}{ Época seca } & \multicolumn{5}{|c|}{ Época úmida } \\
\hline & $\begin{array}{l}0 \\
0 \\
0 \\
\text { n } \\
\text { bo } \\
\text { n } \\
0 \\
0 \\
0 \\
0 \\
0\end{array}$ & $\begin{array}{l}\sum_{i}^{\mathbb{1}} \\
0 \\
0 \\
0 \\
0 \\
0 \\
0 \\
0 \\
0\end{array}$ & 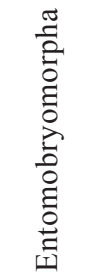 & $\begin{array}{l}\frac{\pi}{7} \\
\stackrel{0}{0} \\
0 \\
0 \\
0 \\
0 \\
0 \\
0\end{array}$ & 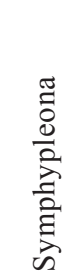 & $\begin{array}{l}0 \\
0 \\
0 \\
\text { in } \\
\text { bo } \\
\text { in } \\
0 \\
0 \\
0 \\
\text { on } \\
\text { in }\end{array}$ & 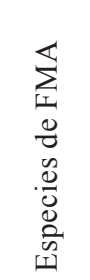 & 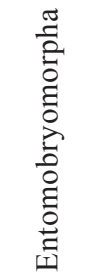 & 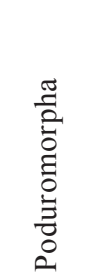 & 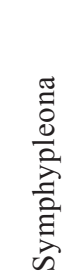 \\
\hline & \multicolumn{10}{|c|}{ Fertilidade do solo } \\
\hline $\mathrm{pH}$ & 0,05 & $-0,69$ & $-0,31$ & $-0,66$ & $-0,60$ & 0,00 & $-0,39$ & 0,02 & 0,31 & 0,43 \\
\hline $\mathrm{Al}+3$ & 0,21 & 0,63 & 0,49 & 0,46 & 0,50 & 0,28 & 0,39 & $-0,30$ & $-0,30$ & $-0,38$ \\
\hline $\mathrm{Ca}+2$ & $-0,01$ & $-0,19$ & $-0,04$ & $-0,34$ & $-0,22$ & $-0,52$ & $-0,19$ & 0,15 & 0,21 & 0,29 \\
\hline $\mathrm{Mg}+2$ & $-0,11$ & $-0,50$ & $-0,51$ & $-0,01$ & $-0,40$ & 0,08 & $-0,14$ & $-0,29$ & $-0,27$ & 0,55 \\
\hline $\mathrm{K}+$ & 0,03 & $-0,23$ & $-0,06$ & 0,09 & 0,06 & 0,09 & $-0,24$ & $-0,52$ & $-0,61$ & 0,57 \\
\hline $\mathrm{P}$ & 0,07 & $-0,29$ & 0,09 & $-0,20$ & $-0,09$ & 0,09 & $-0,13$ & $-0,49$ & $-0,34$ & 0,44 \\
\hline \multirow[t]{2}{*}{$\mathrm{C}$} & 0,30 & $-0,18$ & 0,19 & 0,08 & 0,11 & 0,36 & $-0,20$ & $-0,66$ & $-0,30$ & 0,32 \\
\hline & \multicolumn{10}{|c|}{ Características físicas do solo } \\
\hline Areia grossa & 0,41 & $-0,52$ & 0,03 & $-0,58$ & $-0,60$ & 0,15 & 0,09 & $-0,36$ & 0,00 & 0,44 \\
\hline Areia fina & 0,01 & 0,18 & 0,18 & $-0,23$ & $-0,13$ & $-0,32$ & 0,30 & 0,53 & 0,41 & $-0,43$ \\
\hline Silte & $-0,57$ & $-0,09$ & $-0,75$ & $-0,04$ & $-0,52$ & 0,24 & 0,02 & 0,15 & $-0,17$ & 0,10 \\
\hline Argila & $-0,03$ & 0,33 & 0,24 & $\mathbf{0 , 5 8}$ & $\mathbf{0 , 7 7}$ & $-0,04$ & $-0,25$ & $-0,11$ & $-0,15$ & $-0,13$ \\
\hline Densidade & 0,69 & 0,12 & 0,67 & 0,24 & 0,50 & 0,45 & 0,19 & $-0,70$ & $-0,16$ & $-0,18$ \\
\hline \multirow[t]{2}{*}{ Porosidade } & $-0,79$ & 0,16 & $-0,63$ & 0,05 & $-0,32$ & $-0,53$ & $-0,08$ & $\mathbf{0 , 8 0}$ & 0,17 & 0,11 \\
\hline & \multicolumn{10}{|c|}{ Vegetação } \\
\hline Número de espécies & $-0,45$ & 0,22 & $-0,15$ & $-0,10$ & $-0,09$ & $-0,67$ & $-0,05$ & $\mathbf{0 , 8 2}$ & 0,54 & $-0,14$ \\
\hline Dominância & $-0,50$ & 0,17 & $-0,16$ & $-0,25$ & $-0,09$ & $-0,74$ & $-0,09$ & $\mathbf{0 , 8 2}$ & 0,10 & $-0,23$ \\
\hline Densidade & $-0,73$ & 0,15 & $-0,57$ & 0,36 & $-0,05$ & $-0,53$ & 0,19 & $\mathbf{0 , 8 4}$ & $-0,01$ & $-0,33$ \\
\hline
\end{tabular}

Valores destacados apresentam significância a 5\%; Unidades: $\mathrm{pH}=\mathrm{CaCl}_{2} ; \mathrm{Al}^{3+}, \mathrm{Ca}^{2+}, \mathrm{Mg}^{2+} \mathrm{e} \mathrm{K}^{+}=\mathrm{cmol}_{\mathrm{c}} \mathrm{dm}^{-3} ; \mathrm{P}=\mathrm{mg} \mathrm{dm}{ }^{-3} ; \mathrm{C}=\mathrm{g} \mathrm{dm}^{-3} ; \mathrm{Areia}_{\text {grossa, }} \mathrm{Areia}^{2}$ fina, Silte e Argila $=\mathrm{g} \mathrm{kg}^{-1}$; Densidade do solo $=\mathrm{g} \mathrm{cm}^{-3}$; Porosidade $=\%$; Dominância $=\mathrm{m}^{2} \mathrm{ha}^{-1}$; Densidade $=$ Ind. ha ${ }^{-1}$.

\section{Conclusões}

As comunidades de colêmbolos e de FMA utilizadas como indicadores de degradação, mesmo ocupando diferentes estratos verticais do solo, convergiram para um mesmo resultado, apontando o fragmento P1 como o mais conservado e com pouca diferenciação entre os terços. Além disso, comprovou-se a eficiências desses indicadores, pois se mostraram intimamente influenciados pelas características do solo e da vegetação.

\section{Referências}

AQUINO, A. M.; AGUIAR-MENEZES, E. L.; QUEIROZ, J. M. Recomendações para coleta de artrópodos terrestres por armadilhas de queda (Pitfall-Traps). Seropédica: Embrapa Agrobiologia, 2006. 8 p.

BONFIM, J. A.; VASCONCELOS, R. L. F.; STURMER, S. L.; CARDOSO, E. J. B. N. Arbuscular mycorrhizal fungi in the Brazilian Atlantic forest: A gradient of environmental restoration. Applied Soil Ecology, v. 71, p. 7-14, 2013.

CHAUVAT, M.; PEREZ, G.; PONGE, J. Foraging patterns of soil springtails are impacted by food resources. Applied Soil Ecology, v. 82, p. 72-77, 2014.
DAYNES, C. N.; FIELD, D. J.; SALEEBA, J. A.; COLE, M. A.; MCGEE, P. A. Development and stabilization of soil structure via interactions between organic metter, arbuscular mycorrhizal fungi and plant roots. Soil Biology \& Biochemistry, v. 57, p. 683-694, 2013.

DEEPIKA, S.; KOTHAMASI, D. Soil moisture - a regulator of arbuscular mycorrhizal fungal community assembly and symbiotic phosphorus uptake. Mycorrhiza, v. 24, n. 6, 2014.

EMPRESA BRASILEIRA DE PESQUISA AGROPECUÁRIA EMBRAPA. Corredor ecológico do COMPERJ: um caminho verde para o COMPERJ. Rio de Janeiro: Ecce Design S/C Ltda, 2007. 39 p.

FERREIRA JÚNIOR, W. G.; SCHAEFER, C. E. G. R.; SILVA, A. F. Uma visão pedogeomorfológica sobre as formações florestais da Mata Atlântica. In: MARTINS, S. V. (Ed.). Ecologia de florestas tropicais do Brasil. 2. ed. Viçosa: Ed. UFV, 2012. p. 141-174.

GERDEMANN, J. W.; NICOLSON, T. H. Spores of mycorrhizal endogone species extracted from soil by wet-sieving and decanting. Transactions of the British Mycological Society, v. 46, p. 235-244, 1963.

GOMES, J. B. V.; CURCIO, G. R.; DEDECEK, R. A.; RAMOS, M. R. Atributos dos solos do complexo pertroquímico Comperj em função de variações litotípicas, da paisagem e do uso atual. Pesquisa Florestal Brasileira, v. 34, n. 77, p. 1-11, 2014. 
GREENSLADE, P. Insects of Australia. 2. ed. New York: Melbourne University Press, 1991. p. 252-268.

HEGER, T. J.; IMFELD, G.; MITCHELL, E. A. D. Special issue on "bioindication in soil ecosystems": Editorial note. European Journal of Soil Biology, v. 49, p. 1-4, 2012.

HOPKIN, S. P. Biology of the Springtails (Insecta: Collembola). New York: Oxford University Press, 1997. 340 p.

INSTITUTO NACIONAL DE PESQUISAS ESPACIAIS. FUNDAÇÃO SOS MATA ATLÂNTICA. Atlas dos remanescentes florestais da Mata Atlântica periodo 2011-2012. São Paulo: INPE; SOS Mata Atlântica, 2013.

INTERNATIONAL COLLECTION OF VESICULAR-ARBUSCULAR MYCORRHIZAL FUNGI - INVAM. Disponível em: <http://invam. caf.wvu.edu>. Acesso em: 25 out. 2015.

JEFFERY, S.; GARDI, C.; JONES, A.; MONTANARELLA, L.; MARMO, L.; MIKO, L.; RITZ, K.; PERES, G.; ROMBKE, J.; VAN DER PUTTEN, W. H. European atlas of soil biodiversity. Bruxelas, Bélgica: European Union, 2010. p. 104-105.

MUSCOLO, A.; PANUCCIO, M. R.; MALLAMACI, C.; SIDARI, $\mathrm{M}$. Biological indicators to assess short-term soil quality changes in forest ecosystems. Ecological Indicators, v. 45, p. 416-423, 2014.

PENG, S.; GUO, T.; LIU, G. The effects of arbuscular mycorrhizal hyphal networks on soil aggregations of purple soil in southwest China. Soil Biology \& Biochemistry, v. 57, p. 411-417, 2013.

PEREIRA, C. M. R.; SILVA, D. K. A.; FERREIRA, A. C. A.; GOTO, B. T.; MAIA, L. C. Diversity of arbuscular mycorrhizal fungi in Atlantic forest areas under different land uses. Agriculture, Ecosystems \& Environment, v. 185, p. 245-252, 2014.
RUSEK, J. Biodiversity of Collembola and their functional role in ecosystem. Biodiversity and Conservation, v. 7, p. 1207-1219, 1998.

SCHENK, N. C.; PEREZ, Y. Manual for identification of vesicular arbuscular mycorrhizal fungi. Florida: Synergistic Publications, 1988. $255 \mathrm{p}$.

SMITH, S. E.; READ, D. J. Mycorrhizal symbiosis. London: Academic Press, 2008. 787 p.

TABARELLI, M.; AGUIAR, A. V.; RIBEIRO, M. C.; METZGER, J. P. A conversão da floresta atlântica em paisagens antrópicas: lições para a conservação da diversidade biológica das florestas tropicais. Interciencia, v. 37, n. 2, p. 88-92, 2012.

UHLMANN, A.; BONNET, A.; CURCIO, G. R.; SILVA, A. P.; GONÇALVES, F. L. A.; RESENDE, A. S. A cobertura vegetal das florestas e pastagens. In: PRADO, R. B.; FIDALGO, E. C. C.; BONNET, A. Monitoramento da revegetação do Comperj: etapa inicial. Brasília: Embrapa, 2012. p. 223-244.

ZANGARO, W.; MOREIRA, M. Micorrizas arbusculares nos biomas Floresta Atlântica e Floresta de Araucária. In: SIQUEIRA, J. O.; SOUZA, F. A.; CARDOSO, E. J. B. N., TSAI, S. M. (Eds.). Micorrizas: 30 anos de pesquisas no Brasil. Lavras: UFLA, 2010. p. 279-310.

ZEPPELINI FILHO, D.; BELLINI, B. C. Introdução ao estudo dos Collembola. João Pessoa: Editora da UFPB, 2004. 77 p.

ZEPPELINI, D.; BELLINI, B. C.; CREÃO-DUARTE, A. J.; HERNÁNDEZ, M. I. M. Collembola as bioindicators of restoration in mined sand dunes of Northeastern Brazil. Biodiversity and Conservation, n. 18, p. 1161-1170, 2009.

Contribuição dos autores: Rafael N. Scoriza: Coleta de dados, análise dos dados e escrita do artigo; Maria Elizabeth F. Correia: Elaboração do projeto, análise dos dados e escrita do artigo; Eliane Maria da Silva: Elaboração do projeto e escrita do artigo.

Fonte de financiamento: FAPED.

Conflito de interesse: Os autores declaram não haver conflito de interesse. 